\title{
Neutron Electric Dipole Moment from colored scalars ${ }^{\star}$
}

\author{
Svjetlana Fajfer ${ }^{1,2, a}$ and Jan O. Eeg ${ }^{3, b}$ \\ ${ }^{1}$ Department of Physics, University of Ljubljana, Jadranska 19, 1000 Ljubljana, Slovenia \\ 2 J. Stefan Institute, Jamova 39, P.O.Box 3000, 1001 Ljubljana, Slovenia \\ ${ }^{3}$ Department of Physics, University of Oslo, P.O.Box 1048 Blindern, N-0316 Oslo, Norway
}

\begin{abstract}
We present new contributions to the neutron electric dipole moment induced by a color octet, weak doublet scalar, accommodated within a modified Minimal Flavor Violating framework. These flavor non-diagonal couplings of the color octet scalar might account for an assymmetry of order $3 \times 10^{-3}$ for $a_{C P}\left(D^{0} \rightarrow K^{-} K^{+}\right)-a_{C P}\left(D^{0} \rightarrow \pi^{+} \pi^{-}\right)$ at tree level. The same couplings constrained by this assymmetry also induce two-loop contributions to the neutron electric dipole moment. We find that the direct $\mathrm{CP}$ violating asymmetry in neutral $D$-meson decays is more constraining on the allowed parameter space than the current experimental bound on neutron electric dipole moment.
\end{abstract}

\section{Introduction}

The neutron electric dipole moment (NEDM) plays a special role in current searches of physics beyond the Standard Model (SM). The low energy flavor physics puts extremely tight bounds on possible non-standard model contributions to the NEDM, and in particular special attention has been paid to new sources of CP violation. The NEDM gives a great opportunity to learn about additional sources of CP violation. There are many studies on the NEDM (for a review see [1]). Here we present a study [2] of NEDM within a model suggested by Altmannshofer et al [3] containing a $c \rightarrow u$ flavor changing colored scalar. This model was proposed to explain measurements of the apparent CP asymmetry in $D \rightarrow K^{+} K^{-} / \pi^{+} \pi^{-}$larger than SM expectations. At the time we published our paper[2] the value of the world average CP asymmetry was [4-6]:

$$
\Delta a_{C P}=(-0.329 \pm 0.121) \%,
$$

with $\Delta a_{C P}=a_{K^{+} K^{-}}-a_{\pi^{+} \pi^{-}}$and the definition

$$
a_{f} \equiv \frac{\Gamma\left(D^{0} \rightarrow f\right)-\Gamma\left(\bar{D}^{0} \rightarrow f\right)}{\Gamma\left(D^{0} \rightarrow f\right)+\Gamma\left(\bar{D}^{0} \rightarrow f\right)} .
$$

Now, a new measurement by LHCb presented [7] at this workshop are consistent with zero, and the world average above will be changed.

\footnotetext{
${ }^{\star}$ presented by Jan O. Eeg

a e-mail: svjetlana.fajfer@ijs.si

be-mail: j.o.eeg@fys.uio.no
} 


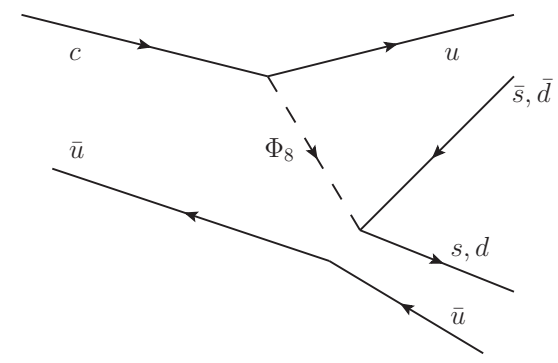

Figure 1. $D \rightarrow P^{+} P^{-}$with $c \rightarrow u$ coupling from colored $\operatorname{scalars}(P=K$ or $\pi)$

Many theoretical studies were performed in order to explain the apparent discrepancy between (1) and an expected SM value, see for example [8-12]. For a a more extensive discussion on New Physics (NP) vs SM physics, we will in this short paper refer to our more extensive paper [2] which has also further references. Some of these studies have explained the observed asymmetry by SM effects [9-12]. For instance, it has been suggested by some authors that some non-perturbative QCD effect like the $\Delta I=1 / 2$ rule in $K \rightarrow \pi \pi$ decays might be responcible for a result like in Eq. (1). Other authors have considered possible NP $[3,8,11]$.

\section{Contributions from a color octet scalar within a modified MFV framework}

New physics have often been parametrized through the Minimal Flavor Violation (MFV) framework [16]. The MFV principle does not forbid appearance of new flavor blind CP violating phases in addition to the unique phase of the CKM matrix. The Flavor Changing Neutral Currents (FCNC) coming from down-like quark sectors are very well known and constrained by experiment.

A viable possibility is to deviate from the generation universality for the color octet couplings to the quark fields, or to modify the original MFV set-up. Thus, the authors of [3] suggested a small deviation from the MFV ansatz by allowing flavor changing $u \leftrightarrow c$ quark interactions with a neutral color octet scalar. Following ref. [3] the interaction Lagrangian may be written as:

$$
\mathcal{L}_{\mathrm{eff}}=G(c \rightarrow u) \bar{u}_{L} T^{A} \Phi^{A} c_{R}+X_{d} \bar{d}_{L} t^{A} d_{R} \Phi^{A}+\text { h.c. },
$$

where the couplings $G(c \rightarrow u)$ and $X_{d}$ are proportional to quark masses:

$$
G(c \rightarrow u) \equiv\left[X_{u}\right]_{12}=\zeta_{u} y_{c} X_{c u} ; \quad X_{c u} \sim V_{c s} V_{u s}^{*} \quad ; \quad X_{d}=\zeta_{d} y_{d}
$$

Here the quantities $\zeta_{u, d}$ are numbers (-to be determined by CP-violation in the charm sector) and $y_{q}=m_{q} / v$, where $v$ is the VEV of the Higgs, and $m_{q}$ is the mass of quark $q$. In this framework the $D^{0}-\bar{D}^{0}$ mixing are not present at tree level. In the case of $D^{0} \rightarrow \pi^{+} \pi^{-}$, the effect is negligible in comparison with the $D^{0} \rightarrow K^{+} K^{-}$amplitude due to the smallness of the down quark mass compared to the bigger mass of the strange quark.

Motivated by the model of [3], we use this tree level effective $\left(c u \Phi_{8}\right)$ coupling bounded by the charm CP asymmetry. Following the work of [3], and using the approriate color factors and Fierz transformations we have found that one can write the asymmetry in Eq. (1), based on (3) as

$$
\Delta a_{C P}=\frac{2}{9} \frac{\zeta^{2}}{M_{\Phi}^{2}} m_{K}^{2} C_{R G E} C_{H} .
$$


Here we have assumed a maximal CP-violating phase $\Phi_{f}$ and and a maximal strong phase $\delta_{f}$. Furthermore, $C_{R G E}$ denotes the factor which includes running of the Wilson coefficient $\left(C_{R G E}=0.85\right.$, for the running from the scale $M_{\Phi} \sim 1 \mathrm{TeV}$ down to the charm scale $m_{c}$ ), while $C_{H}$ stands for a possible enhancement of the hadronic matrix elements, assumed to be as large as $C_{H} \simeq 3$ in comparison with the naive factorization estimate leading to $C_{H}=1$ as explained in [3]. We denote $\zeta^{2}=\zeta_{u} \zeta_{d}$, which appears in the expressions for both NEDM and $\Delta a_{C P}$.

\section{NEDM within the SM}

The NEDM has been studied for many years[1], both within and beyond the SM. The effective Lagrangian for a dipole moment of a fermion $f$ has the generic form

$$
\mathcal{L}_{\mathrm{eff}}=\frac{i}{2} d_{f} \bar{f} \sigma_{\mu \nu} F^{\mu v} \gamma_{5} f
$$

where $d_{f}$ is the EDM of the fermion, $f$ is the fermion field, $F^{\mu v}$ is the electromagnetic field and $\sigma_{\mu \nu}=$ $i\left[\gamma_{\mu}, \gamma_{v}\right] / 2$. Contributions to the NEDM might come from EDMs of single quarks, and contributions due to interplay of quarks within the neutron. In the valence approximation, the contributions to NEDM from EDMs of single quarks $\left(d_{q}\right)$ are given by

$$
d_{n}=\frac{4}{3} d_{d}-\frac{1}{3} d_{u} .
$$

The existing experimental bound from ref. [13] for the NEDM is

$$
d_{n}^{\exp } \leq 2.9 \times 10^{-26} e \mathrm{~cm}
$$

To obtain an EDM within the SM, two weak interactions are needed, and at least one of these must be a (flavor non-diagonal) penguin-like interaction. The EDMs of single quarks, which are three loop diagrams with double Glashow-Iliopoulos-Maiani(GIM)-cancellations and at least one gluon exchange, are of order $\alpha_{s} G_{F}^{2}$ and proportional to quark masses and an imaginary CKM factor. However, in the SM, EDMs of single quarks were found to be very small, and gave a NEDM of order $10^{-34} e$ $\mathrm{cm}[1,2]$.

It was shown that mechanisms with interplay of weak and strong interactions give a contributions bigger than those from single quarks. Typically, such amplitudes were written as baryon poles with two weak interactions, an ordinary $\mathrm{W}$-exchange and a CP-violating penguin interaction, with for instance a negative parity strange baryon as intermediate baryon, and a soft photon emitted from somewhere. There were also contributions due to amplitudes at baryonic/mesonic level where the photon was emitted from an intermediate pion or kaon within a chiral loop. Within such mechanisms, the $d_{n} / e$ was estimated to be of order $10^{-33}$ to $10^{-31} \mathrm{~cm}[1,2]$.

It was pointed out that if the pole diagrams were interpreted at quark level it would correspond to a "diquark mechanism" [14]. This means a CP-violating two loop diagram for the quark process $u d \rightarrow d u \gamma$. Here the contribution was obtained in terms of a two loop factor proportional to $\alpha_{s} G_{F}^{2}$, and a CKM factor. Such diagrams lead to an eight dimensional operator for the NEDM. The obtained result is $d_{n} / e \sim 10^{-33}$ to $10^{-32} \mathrm{~cm}$. Within this mechanism the NEDM is suppressed by a small hadronic matrix element, but had logarithmic GIM instead of power-like, compensating for hadronic suppression.

The operator in (6) has dimension five and it is the lowest dimension operator in SM, if the $\theta$ problem in QCD is rotated away as described in ref. [1]. If the electromagnetic field is replaced by 


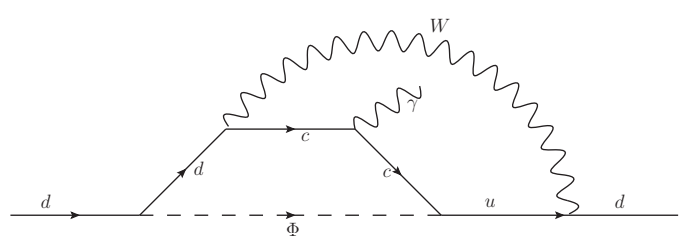

Figure 2. Two loop diagram for an EDM of a $d$-quark

gluonic field $G_{\mu \nu}^{a}$ then one has also colored EDMs of the same dimension. In the study of the NEDM usually the lowest dimensional operators were considered [1]. Recently, it has been pointed out that higher dimensional operators might lead to rather important contributions to NEDM, and that that CP violation in charm decays can induce an increase of the NEDM, if these higher dimensional operators are included [15].

There are other mechanisms for EDMs as the well-known Weinberg operator, and also the socalled Barr-Zee mechanism.

It should also be noted that several authors have recently considered the NEDM within frameworks beyond the SM ( see for instance refs. [17-19]).

\section{Two-loop contributions to EDM}

Motivated by the explanation of direct CP violation in decay of the neutral $D$-meson by the presence of a color octet, we investigate the impact of the imaginary couplings of quarks to the color scalars on the NEDM. Then we find that there is a new two-loop contribution, which we consider in the following subsection. We also find that these couplings induce higher dimensional operators present in NEDM. It is important to notice that such couplings cannot affect any other low energy observable, as already discussed in ref. [3].

Two-loop contributions are induced by the presence of the $c \rightarrow u$ flavor changing color octet couplings described in the modified MFV framework above. We have found that the two loop contribution in Fig. 2 induces a dimension 5 electric dipole operator for the $d$-quark which might be written as an effective Lagrangian in the following way:

$$
\mathcal{L}_{1}(d \rightarrow d \gamma)_{\Phi}=K\left(\bar{d}_{L} \sigma \cdot F d_{R}\right)
$$

The quantity $K$ is given by

$$
K=C_{3}\left[g_{W}^{2} V_{u d} V_{c d}^{*} G(c \rightarrow u) X_{d}\right] 2 m_{c} e_{c} I_{2-l o o p},
$$

where $C_{3} \equiv<T^{A} T^{A}>=4 / 3$ is a color factor and the leading logarithmic approximation of the two loop integral is:

$$
I_{2-\text { loop }} \simeq\left(\frac{1}{16 \pi^{2}}\right)^{2} \frac{1}{M_{\Phi}^{2}}\left(\left[\ln \frac{M_{\Phi}^{2}}{m_{c}^{2}}\right]^{2}-\left[\ln \frac{M_{W}^{2}}{m_{c}^{2}}\right]^{2}\right) .
$$

There is also a contribution from the crossed diagram with the result:

$$
\mathcal{L}_{2}(d \rightarrow d \gamma)_{\Phi}=K^{*}\left(\bar{d}_{R} \sigma \cdot F d_{L}\right),
$$




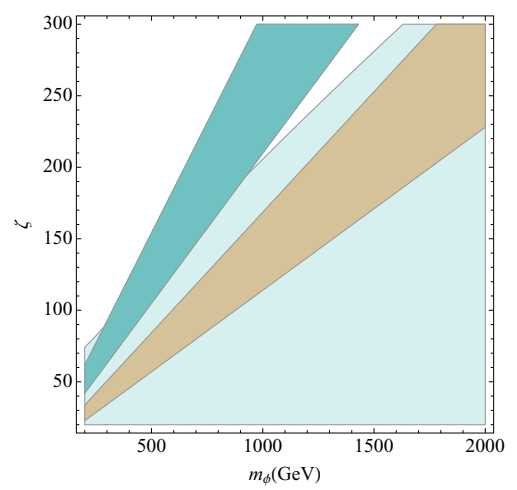

Figure 3. Regions in the $\zeta-M_{\Phi}$ plane compatible with the data on $\Delta a_{C P}$ (dark green, $C_{H}=1$ and pale brown for $C_{H} \simeq 3$ ) and on the current experimental lower bound on $N E D M$ (pale green).

such that there will be an EDM of the $d$-quark equal to $\left(d_{n}\right)_{2-\text { loop }}^{\Phi}=2 \operatorname{Im}(K)$. Note that these diagrams appear due to the presence of appropriate chiralities in the interacting Lagrangian (3). The EDM of the $u$-quark is suppressed by extra factors of small masses. We have checked that the opposite chirality of the the scalars lead to two-loop helicity suppressed amplitudes.

Assuming that our result for the $d$ quark electric dipole moment gives arise to NEDM as given in (7), we bound our new contribution to NEDM by the current experimental result given in Eq. (8). Both constraints are presented on Fig. 3.

In the case that the mass of color octet is bounded in the TeV regime, the parameter $\zeta^{2}$ should be scaled accordingly. We have checked that even for a mass of $M_{\Phi} \geq 1.86 \mathrm{TeV}$, perturbativity is still valid for the couplings in (3). That means that one can safely use the effective Lagrangian (3) for this purpose. We point out that the loop diagram in Fig. 2 is finite due to the chiral structure of (3), and that we have only given the relevant leading logarithmic result. Of course the leading log. result in (11) will be modified when taking into account higher orders by means of renormalization group techniques, but at the present stage we use this result. For fixed asymmetry - i.e. $\zeta^{2} / M_{\Phi}^{2}$ fixed - we obtain the relation

$$
\left(d_{n} / e\right)_{2-l o o p}^{\Phi} \simeq\left(\frac{\lambda^{2} m_{d}}{8 \pi^{4}}\right) \frac{M_{W}^{2} m_{c}^{2}}{v^{4} m_{K}^{2}} \frac{\Delta a_{C P}}{C_{R G E} C_{H}}\left(\left[\ln \frac{M_{\Phi}^{2}}{m_{c}^{2}}\right]^{2}-\left[\ln \frac{M_{W}^{2}}{m_{c}^{2}}\right]^{2}\right) .
$$

Numerically, we obtain the range

$$
\left(d_{n} / e\right)_{2-l o o p}^{\Phi} \simeq(1.0-2.3) \times 10^{-26} \mathrm{~cm},
$$

for $M_{\Phi}$ in the range $400 \mathrm{GeV}$ to $2 \mathrm{TeV}$, assuming $C_{H} \simeq 3$. It is interesting that for $C_{H}=1$, we would get $d_{n}$ three times bigger and violate the experimental bound in Eq.(8)! Let us comment that this result should also be valid for other flavor changing $(c u \Phi)$ colored scalar (triplet, sextet) couplings, with appropriate color factor replacements.

We have also considered the di-quark mechanism obtained from one loop diagrams for $W^{-} u \rightarrow d \gamma$ and $W^{+} d \rightarrow u \gamma$, and where the $W$-boson is attached to left-handed $u \rightarrow d$ and $d \rightarrow u$ currents. These contributions are of same type as in [14] and of same numerical order as within the SM (For further details, see our paper [2]). 
EPJ Web of Conferences

\section{Acknowlwedgements}

JOE is supported in part by the Norwegian research council. Work of SF is partially supported by Slovenian research agency ARRS.

\section{References}

[1] M. Pospelov and A. Ritz, Annals Phys. 318 (2005) 119 [hep-ph/0504231].

[2] S. Fajfer and J.O. Eeg, Phys.Rev. D89, 095030, 2014 [arXiv:1401.2275[hep-ph]]

[3] W. Altmannshofer, R. Primulando, C. -T. Yu and F. Yu, JHEP 1204 (2012) 049 [arXiv:1202.2866 [hep-ph]].

[4] http://www.slac.stanford.edu/xorg/hfag/

[5] R. Aaij et al. [LHCb Collaboration], Phys. Rev. Lett. 108 (2012) 111602 [arXiv:1112.0938 [hepex]].

[6] T. Aaltonen et al. [CDF Collaboration], Phys. Rev. D 85 (2012) 012009 [arXiv:1111.5023 [hepex]].

[7] M. Martinelli, talk on this workshop

[8] G. Isidori, J. F. Kamenik, Z. Ligeti and G. Perez, Phys. Lett. B 711 (2012) 46 [arXiv:1111.4987 [hep-ph]].

[9] H. -Y. Cheng and C. -W. Chiang, Phys. Rev. D 86 (2012) 014014 [arXiv:1205.0580 [hep-ph]].

[10] J. Brod, Y. Grossman, A. L. Kagan and J. Zupan, JHEP 1210 (2012) 161 [arXiv:1203.6659 [hep-ph]].

[11] A. Lenz, arXiv:1311.6447 [hep-ph].

[12] P. Santorelli, talk at this workshop

[13] C. A. Baker, D. D. Doyle, P. Geltenbort, K. Green, M. G. D. van der Grinten, P. G. Harris, P. Iaydjiev and S. N. Ivanov et al., Phys. Rev. Lett. 97 (2006) 131801 [hep-ex/0602020].

[14] J.O. Eeg, and I. Picek, Nucl.Phys. B244 (1984) 77

[15] T. Mannel and N. Uraltsev, JHEP 1303 (2013) 064 [arXiv:1205.0233 [hep-ph]].

[16] G. D’Ambrosio, G. F. Giudice, G. Isidori and A. Strumia, Nucl. Phys. B 645 (2002) 155 [hep$\mathrm{ph} / 0207036]$.

[17] J. Brod, U. Haisch, and J. Zupan, JHEP 1311 (2013) 180, [arXiv:1310.1385 [hep-ph]]

[18] Xiao-Gang He, Chao-Jung Lee, Siao-Fong Li, and J. Tandean, arXiv:1404.4436 [hep-ph]]

[19] M. Gorbahn and U. Haisch, arXiv: 1404.4873 [hep-ph]] 